\title{
Health-related quality of life and
}

\section{functional status in end-stage COPD: a longitudinal study}

\author{
J.M. Habraken*,\#, W.M. van der Wal ${ }^{\tau,+}$, , G. ter Riet*, E.J.M. Weersink ${ }^{\S}$, \\ F. Toben ${ }^{f}$ and P.J.E. Bindels**
}

ABSTRACT: Since there is still a dearth of information about the end stage of chronic obstructive pulmonary disease (COPD), the main aim of this study was to examine the development of healthrelated quality of life (HRQOL) and functional status over time in COPD patients in Global Initiative for Chronic Obstructive Lung Disease (GOLD) stage IV.

82 Dutch COPD patients completed the St George's Respiratory Questionnaire (SGRQ) for HRQoL and the Groningen Activities for Daily Living Restriction Scale (GARS) for functional status every 3 months during the year following enrolment. Survival was followed up to 5 yrs after enrolment. Data were analysed by stratifying the study population into severity subgroups according to the lowest, intermediate and highest tertile of SGRQ and GARS at baseline. Outcome measures were change in SGRQ and GARS scores over time and survival time.

In the majority of patients, scores on the SGRQ and GARS declined gradually over time. In the subgroup of 32 patients that died within 2 yrs of enrolment, these scores also declined gradually, without steep deteriorations.

In patients with end-stage COPD, HRQoL and functional status deteriorated gradually over time, indicating that clinicians did not gain much additional support for differentiating the end stage of COPD by considering HRQoL and functional status using the SGRQ and GARS.

KEYWORDS: Activities of daily living, chronic obstructive pulmonary disease, longitudinal studies, palliative care, quality of life

hronic obstructive pulmonary disease (COPD) is one of the most important causes of morbidity and mortality worldwide $[1,2]$. COPD is a progressive illness, characterised by acute episodes of ill health (exacerbations). Although it is well known that COPD is a potentially fatal disease, there is still a dearth of information about the end stage of the disease. Patients in the end stage of COPD usually experience and survive several severe exacerbations, but any one may prove to be the fatal one $[3,4]$. Therefore, the end stage of COPD is hard to define $[5,6]$. Because of this unpredictable trajectory of the end stage of COPD, patient-physician communication about palliative and end-of-life care is unlikely to occur [7-9] and, as a consequence, the death of a COPD patient may feel sudden and unexpected for both patients and family members [10].

Studies on the final years of patients with advanced COPD have shown that these are characterised by uncontrolled symptoms, such as intolerable dyspnoea [10-14], and high levels of anxiety [10, 14, 15], depression $[10,14,16]$ and social isolation $[13,17,18]$. It is also known that, despite these uncontrolled symptoms, patients in the end stage of COPD have limited access to specific palliative care services when compared to, for example, patients suffering from advanced lung cancer [11, 19, 20]. Crosssectional studies have shown that health-related quality of life (HRQoL) is low in these patients, even compared to advanced lung cancer patients [11, 19, 21]. ClaEsSENS et al. [20] state, in their report on the results of the Study to Understand Prognosis and Preferences for Outcomes and Risks of Treatments (SUPPORT), that despite similar preferences about end-of-life care, hospitalised patients with COPD were more likely to have mechanical ventilation, tube feeding and cardiopulmonary resuscitation, compared with patients with lung cancer. It is thus recognised that patients in the end stage of COPD have care needs that are not currently being met [11, 18, 22].

A few studies have examined the course of HRQoL by using longitudinal measures [23, 24]. These studies, however, focused on patients with

\section{AFFILIATIONS}

*Dept of General Practice,

'Dept of Clinical Epidemiology, Biostatistics and Bioinformatics,

${ }^{\S}$ Dept of Pulmonology, Amsterdam Medical Center, Amsterdam,

${ }^{\#}$ Academic Research Centre for Health and Social Care, Tilburg University, Tilburg,

${ }^{+}$Dept of Biostatistics, Julius Center, University Medical Center, Utrecht,

${ }^{f}$ Dept of Pulmonology, and

**Dept of General Practice, Erasmus Medical Center, Rotterdam,

The Netherlands.

CORRESPONDENCE

J.M. Habraken

Clinical Methods and Public Health,

Dept of General Practice, Academic

Medical Center

University of Amsterdam

Meibergdreef 15

1105 AZ

Amsterdam

The Netherlands

E-mail: jolanda_habraken@

yahoo.com

Received:

Sept 222009

Accepted after revision:

May 292010

First published online:

June 072010 
moderate-to-severe COPD, and not on patients in the end stage of COPD. It would, therefore, be important to get a better understanding about the clinical course of the end stage of COPD, as expressed by the patients' HRQoL. If COPD patients in the end stage report significant changes in their perceived HRQoL, this may provide clinicians with tools for improving palliative care provision at the end of life. This would be aimed specifically at treating unmet care needs, and the timing of communication about palliative and end-of-life decisions, benefitting both patients and their informal caregivers.

Our main aim was, therefore, to examine the development of HRQoL and functional status over time in patients in the end stage of COPD. We set out to explore changes in HRQoL and functional status over time, by using repeated measurements over $1 \mathrm{yr}$ in COPD patients in Global Initiative for Chronic Obstructive Lung Disease (GOLD) stage IV [25].

\section{METHODS}

\section{Patients}

Between March 2004 and November 2006, we included Dutch COPD patients in GOLD stage IV. The inclusion criteria were a forced expiratory volume in $1 \mathrm{~s}(\mathrm{FEV} 1)<30 \%$ predicted and age $\geqslant 60$ yrs. Patients were identified from the medical records of the outpatient clinics of four participating hospitals and one centre specialising in asthma and COPD, all situated in The Netherlands. Because data at baseline were compared to advanced lung cancer patients (described in detail elsewhere [21]), coexistence of stage IIIb or IV lung cancer was an exclusion criterion. Patients were asked to complete a selfadministered questionnaire at baseline and every 3 months over a period of $1 \mathrm{yr}$, with a maximum of four measurements per patient, including the baseline measurement. The data were collected by postal questionnaires. After 5 yrs, general practitioners were contacted for survival information. All patients provided written informed consent. The research ethics committee of the Amsterdam Medical Center (Amsterdam, The Netherlands) reviewed the complete study protocol and decided that, as the study consisted of interviews and questionnaires only, it did not require formal ethics review.

\section{HRQoL}

All patients completed the disease-specific St George's Respiratory Questionnaire (SGRQ) repeatedly over 1 yr [26]. This questionnaire has four domains: symptoms, impacts, activities and a total score. Scores are transformed into percentage points ranging $0-100 \%$; higher scores indicate a worse HRQoL. A difference in SGRQ score of four is considered to be clinically significant [27].

For a subjective measurement of functional status, patients also completed the Groningen Activities of Daily Living Restriction Scale (GARS) questionnaire repeatedly over $1 \mathrm{yr}$ [28]. The GARS measures activities of daily living (ADL) (personal care; range 11-33 points) as well as instrumental ADL (IADL)

\section{TABLE 1}

Demographic characteristics and data related to lung function, treatment, dyspnoea, St George's Respiratory Questionnaire (SGRQ) and Groningen Activities of Daily Living Restriction Scale (GARS) score for the total group by SGRQ and GARS scores at baseline

\begin{tabular}{|c|c|c|c|c|c|c|}
\hline & \multicolumn{3}{|c|}{$\begin{array}{c}\text { Severity based on SGRQ at } \\
\text { baseline }\end{array}$} & \multicolumn{3}{|c|}{$\begin{array}{c}\text { Severity based on GARS ADL score at } \\
\text { baseline }\end{array}$} \\
\hline & Low & Intermediate & High & Low & Intermediate & High \\
\hline \multicolumn{7}{|l|}{ HRQoL functional status mean } \\
\hline SGRQ score at baseline & $47.3 \pm 8.8$ & $67.1 \pm 4.3$ & $81.0 \pm 6.3$ & $52.6 \pm 13.9$ & $67.0 \pm 11.6$ & $75.3 \pm 11.1$ \\
\hline GARS score at baseline & $28.6 \pm 6.8$ & $35.1 \pm 6.4$ & $39.2 \pm 5.8$ & $25.8 \pm 3.6$ & $33.8 \pm 2.0$ & $42.6 \pm 3.3$ \\
\hline \multicolumn{7}{|l|}{ Demographics } \\
\hline Patients n & 27 & 27 & 28 & 27 & 25 & 30 \\
\hline Age yrs & $69.3 \pm 7.2$ & $69.8 \pm 6.4$ & $69.3 \pm 6.9$ & $66.6 \pm 6.2$ & $70.8 \pm 6.6$ & $70.9 \pm 6.8$ \\
\hline $\mathrm{BMI} \mathrm{kg} \cdot \mathrm{m}^{-2}$ & $23.4 \pm 3.9$ & $23.3 \pm 2.6$ & $22.0 \pm 4.6$ & $23.8 \pm 3.4$ & $21.4 \pm 3.8$ & $23.3 \pm 3.9$ \\
\hline Females & $10(37)$ & $7(26)$ & $11(39)$ & $10(37)$ & $9(36)$ & $9(30)$ \\
\hline Living alone & $9(33)$ & $4(15)$ & $8(29)$ & $7(26)$ & $9(36)$ & $5(17)$ \\
\hline \multicolumn{7}{|l|}{ Lung function } \\
\hline FEV $1 \%$ pred & 25 (24-28) & $25(22-27)$ & 25 (22.3-27) & $26(24-28)$ & $26(24-28)$ & $25(20-26)$ \\
\hline \multicolumn{7}{|l|}{ Treatment } \\
\hline Admitted to hospital in the previous year & $12(44)$ & $13(48)$ & $16(57)$ & $12(44)$ & $13(52)$ & $16(53)$ \\
\hline Ever attended pulmonary rehabilitation therapy & $10(37)$ & $15(56)$ & $13(46)$ & $10(37)$ & $10(40)$ & $18(60)$ \\
\hline Current use of long-term oxygen therapy & $10(37)$ & $14(52)$ & $13(46)$ & $6(22)$ & $13(52)$ & $18(60)$ \\
\hline \multicolumn{7}{|l|}{ Dyspnoea } \\
\hline MRC dyspnoea scale & $3(1-4)$ & $4(4-5)$ & $5(5-5)$ & $4(2-4)$ & $4(4-5)$ & $5(5-5)$ \\
\hline
\end{tabular}

Data are presented as mean $\pm \mathrm{SD}, \mathrm{n}(\%)$ or median (interquartile range), unless otherwise stated. Higher scores on SGRQ and GARS indicate increasing severity. ADL: activities of daily living; HRQoL: health-related quality of life; BMI: body mass index; FEV1: forced expiratory volume in 1 s: \% pred: \% predicted; MRC: Medical Research Council. 


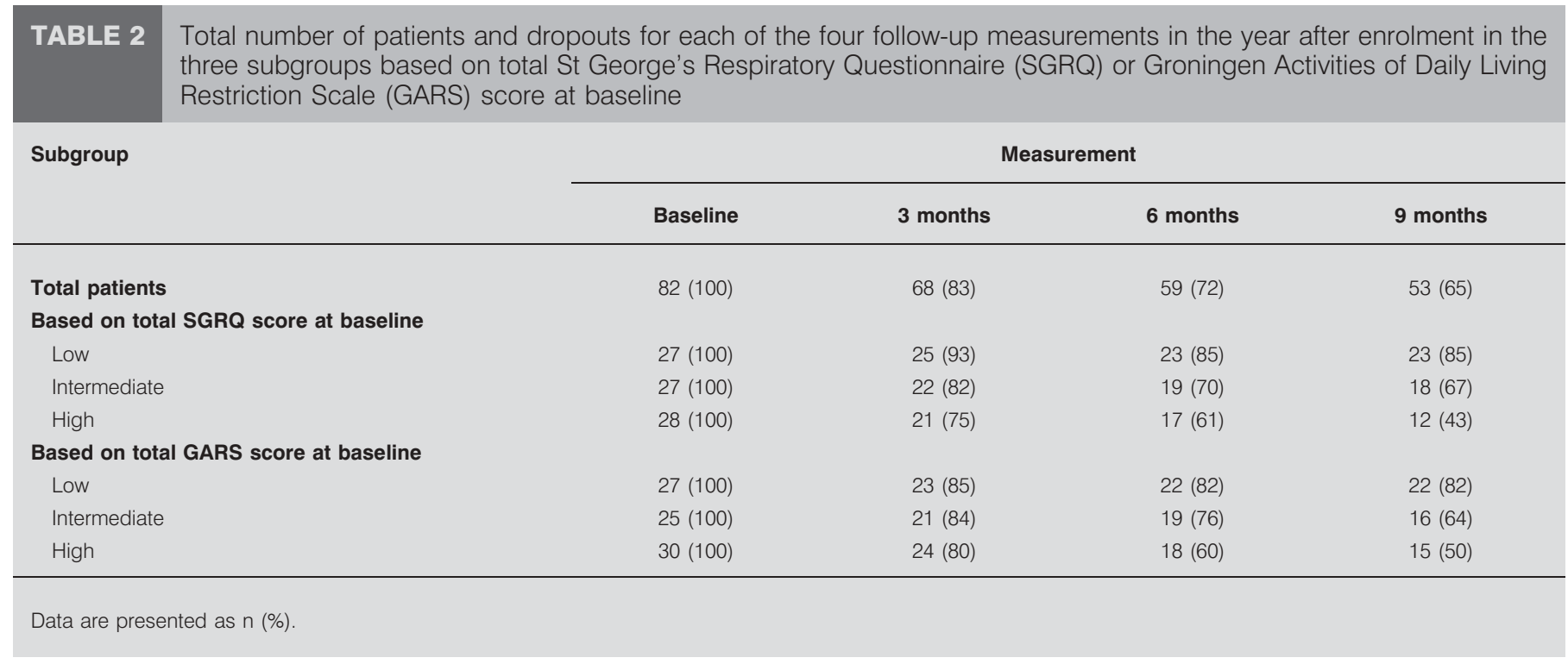

(domestic activities; range 7-28 points). The total score has a range of 18-54. Higher scores indicate worse daily functioning in all domains. Because of the important role of dyspnoea, the Medical Research Council dyspnoea scale was also included [29]. The scale ranges 1-5, where higher scores indicate worse daily functioning due to breathlessness.

\section{Statistical analysis}

Definition of severity subgroups

Our main aim was to examine the development of HRQoL over $1 \mathrm{yr}$ in or near the end stage of the disease. At baseline, there were considerable differences between patients, both in the GARS and the SGRQ. To deal with this high interindividual variability, we stratified the study population into severity subgroups according to the lowest, intermediate and highest tertile of SGRQ and GARS at baseline. These strata were not predefined by the scales, but were developed from the data. By stratifying on these subgroups, we corrected for possible bias due to selective dropout (see later).

\section{Statistical modelling}

We investigated the development of SGRQ and GARS over time using mixed models. We regressed both the total scores and subscales of SGRQ and GARS on time since baseline and severity subgroup use random intercepts and slopes. These models allowed individual patients to start at different scores at baseline and allowed their linear trend over time to vary individually. In addition, we included interactions between time and severity subgroup to assess if the courses differed between the three subgroups. Using these models, we investigated whether a possible decline in SGRQ and GARS over time differed according to baseline SGRQ and GARS, respectively.

\section{Selective dropout}

Dropout during the study was summarised numerically in a table and survival was explored using Kaplan-Meier curves. We examined the possibility of bias in our description of the development of SGRQ and GARS due to selective dropout (due to rapid deterioration or death); individuals with poorer SGRQ or GARS might drop out sooner than individuals with better SGRQ or GARS. Therefore, during the course of the study, individuals remaining in the study could be relatively healthy. The development of SGRQ and GARS in this subselection of relatively healthy individuals would not be representative for the development of SGRQ and GARS in the study population.

Using univariate Cox proportional hazards models, it was confirmed that both SGRQ and GARS (including all subdomains) were associated with dropout due to deterioration or death. However, the effects of SGRQ and GARS on dropout disappeared by including severity subgroup in the models (either low, intermediate or high SGRQ at baseline, or low, intermediate or high GARS at baseline, respectively). This implies that by stratifying by severity subgroup, we adjusted for selective dropout. This stratification was done in two ways: first, by plotting the development of GARS and SGRQ over time separately for each subgroup; secondly, by including subgroup as a covariate in the mixed models described above.

\section{Imputation}

Of the total number of 262 measurements that were taken in the 82 patients, there were no missing values in the SGRQ domains. However, for the total GARS, GARS ADL and GARS IADL domains, there were 17, 12 and 13 missing values, respectively. These missing values were imputed using mixed models, regressing the GARS scales on time since inclusion and all baseline covariates, and allowing for random intercepts and slopes.

\section{RESULTS}

A total of 82 COPD patients in GOLD stage IV was included in the study. The median time between measurements was 111 days (10th and 90th percentile were 97 and 140 days, respectively), which is somewhat more than the planned 3 months (91 days). The total number of measurements was 262. By stratifying the study population according to disease 


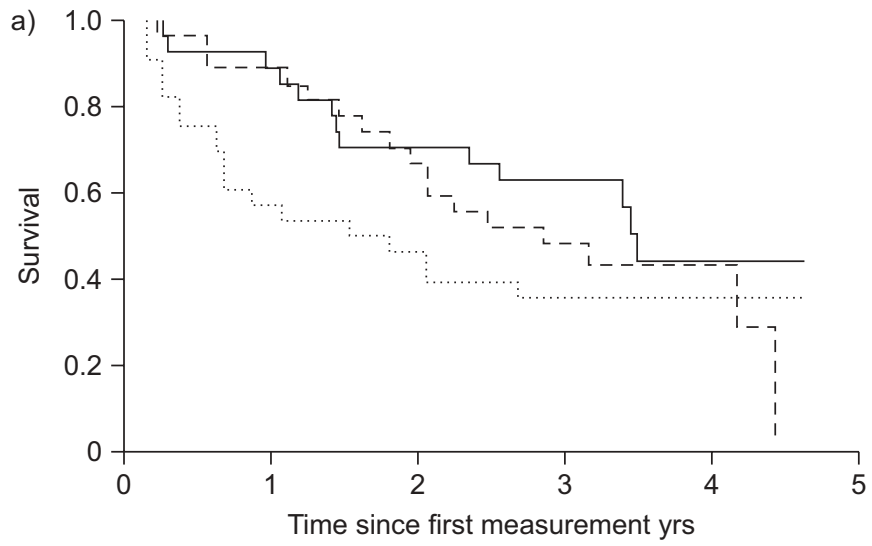

b)

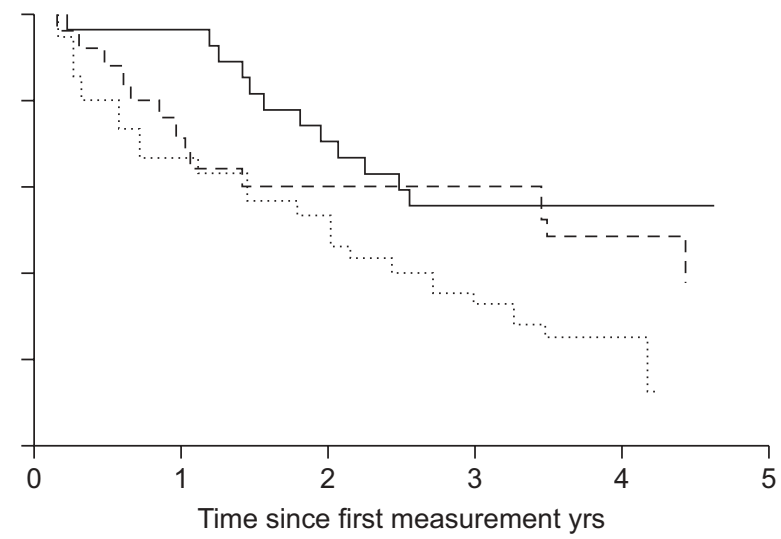

FIGURE 1. Kaplan-Meier curves of cumulative survival within the first 5 yrs following data entry for low- (-), intermediate- (---) and high-severity (..........) subgroups, based on total a) St George's Respiratory Questionnaire and b) Groningen Activities of Daily Living Restriction Scale scores at baseline.

severity, the study population was divided into subgroups in two different ways: one division based on the mean SGRQ scores at baseline and the other based on the mean GARS scores at baseline. Table 1 summarises baseline characteristics of the study population separately for each severity subgroup. There were no important differences between the subgroups with respect to demographics and lung function. However, the treatment characteristics were consistently higher in the highseverity group than in the low-severity group, indicating that patients in the high-severity group were treated more often that patients in the low-severity group.

Table 2 shows the number of patients in the study population and the number of dropouts for each of the four follow-up measurements. Of the 29 patients who dropped out of the study, 23 died during, or within the 5 yrs following, the study period. Two patients dropped out because of emotional problems with the questionnaires. Four patients were lost to follow-up. Higher severity seems to be associated with a higher dropout rate. This means that patients who entered the study with worse quality of life and functional status dropped out more than patients who entered the study with more favourable quality of life and functional status scores.

A higher probability of survival in the low-severity subgroups was seen in Kaplan-Meier curves for the six subgroups (fig. 1). Figure 1 shows the cumulative survival during the 5 yrs from the start of the study, for all severity subgroups. 2 yrs after entering the study, patients in the high-severity subgroup had a probability of survival of 0.46 (95\% CI $0.31-0.69)$ based on SGRQ and 0.53 (95\% CI 0.38-0.75) based on GARS, compared to a probability of survival of 0.70 (95\% CI 0.55-0.90) based on both SGRQ and GARS of the low-severity subgroup at the same time-point.

Boxplots of the three subgroups of SGRQ and GARS are shown in figures 2 and 3, respectively. In figure 2, development of the SGRQ over time is shown for each of the severity subgroups. In the activity domain (fig. 2b), there appears to be a ceiling effect, i.e. patients scored so highly that there was no more room for them to score even higher (worse) in this domain. In the other domains, no clear ceiling effect is apparent, although the upper limits of the confidence intervals of the high-severity subgroups are close to the maximal score of $100 \%$. What is most apparent in figure 2 is that, within each severity subgroup, the courses of the SGRQ domains show little difference over time. In the impacts (fig. 2c) and total (fig. 2d) domains, there was a slight shift towards a higher score on the fourth measurement, but this shift was small and only visible in the subgroup that entered the study with the most favourable HRQoL.

Figure 3 shows the development of the GARS scores. The same shallow and gradual pattern as that in figure 2 can be seen. In figure 3, the upper limits of the confidence intervals of the high-severity subgroup also indicate some ceiling phenomena, in the IADL and total domains in particular.

In figure 4, the total SGRQ and GARS scores are presented over time for those patients who died within 2 yrs of the start of the study $(n=32)$. With very few exceptions, figure 4 shows that there are no sudden changes in mean scores at the end of life.

Figure 5 shows representative plots of the development of total SGRQ and GARS scores for six individual patients. These individual plots are an illustration of the variability within our population. Figure $5 \mathrm{a}-\mathrm{c}$ shows score patterns that are typical for the overall trends in figures 2 and 3 . Conversely, figure $5 \mathrm{~d}-\mathrm{f}$ illustrates that, in some cases, considerable variation in HRQoL and functional status over time may occur. The differences in scores within these patients are substantial, given the fact that a difference of four in SGRQ scores is considered to be clinically significant [27].

Table 3 shows the fixed intercepts and fixed 1-yr increase in SGRQ and GARS scores from the mixed models described above, for all domains and subgroups separately.

Predicted 1-yr increase in SGRQ and GARS score was significant in the low- and intermediate-severity subgroups, except for the SGRQ symptoms domain. Predicted increases were largest in the low-severity subgroups. In SGRQ, the low-severity subgroup showed a statistically and clinically significant increase of $5.6(95 \%$ CI $2.5-8.8)$ in the activity domain, 7.0 (95\% CI 3.0-11.1) in the impacts domain and 6.0 (95\% CI 3.2-8.8) in the total domain. In GARS, the low-severity 


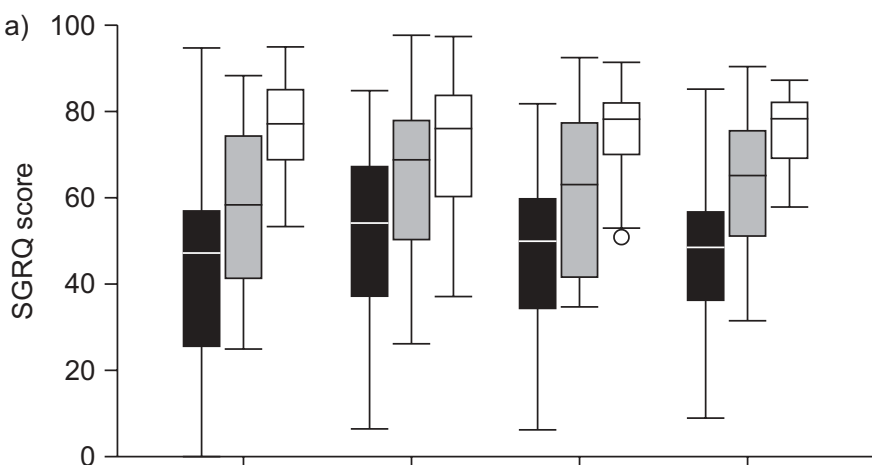

b)
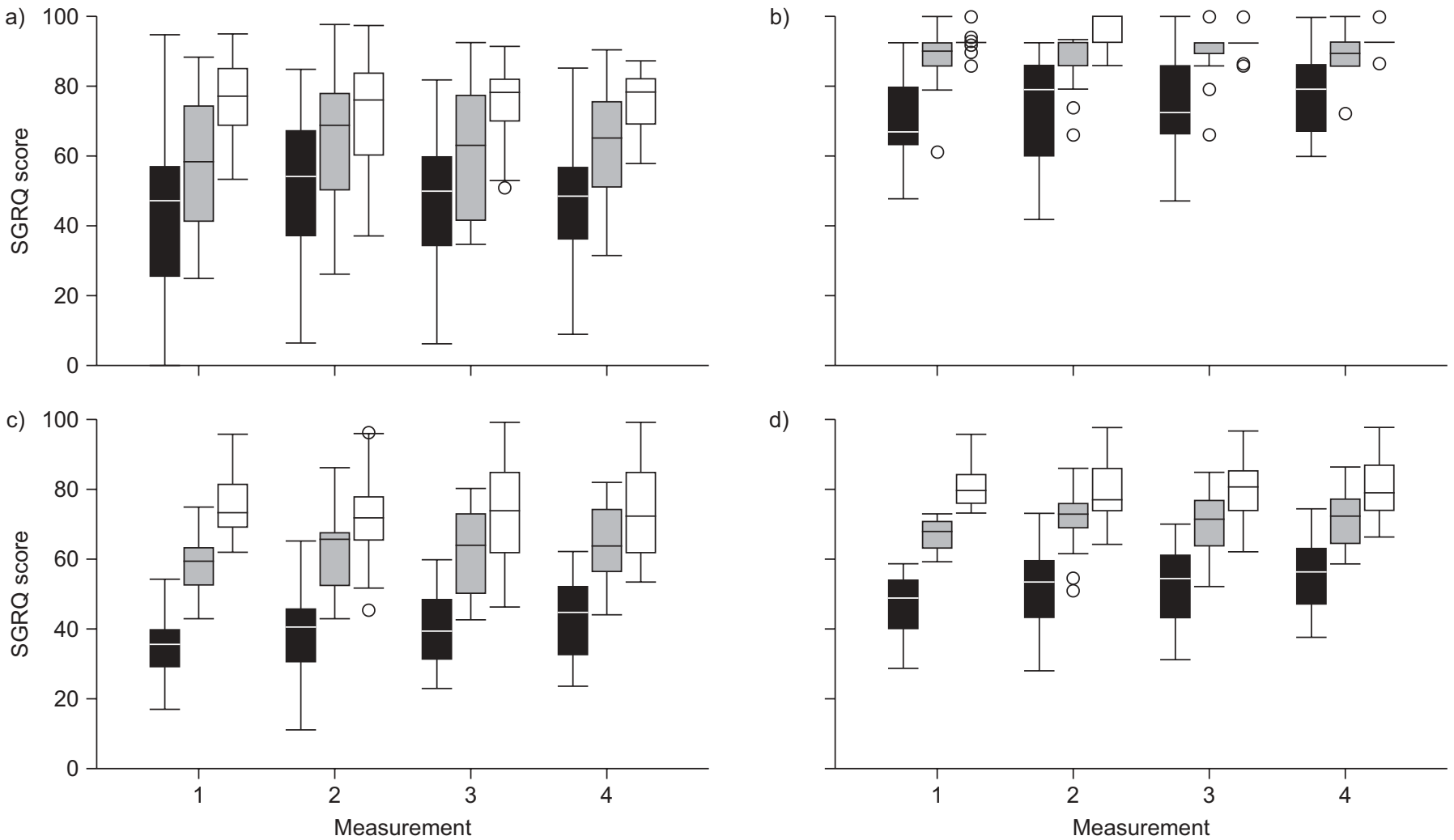

d)

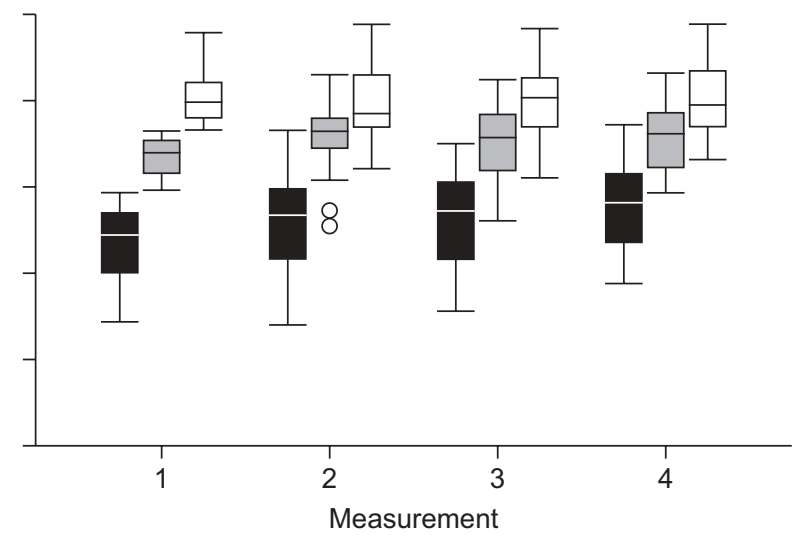

FIGURE 2. Boxplots of the a) symptoms, b) activity, c) impacts and d) total domains of the St George's Respiratory Questionnaire (SGRQ), at 0-9 months after data entry, by severity subgroup (based on total SGRQ score at baseline). Boxes represent the interquartile range and whiskers represent the range. -: median; $\bigcirc:$ outliers. low severity; : $:$ : intermediate severity; $\square$ : high severity.

subgroup showed a statistically significant increase of $1.0(95 \%$ CI 0.2-1.9) in the ADL domain, 0.9 (95\% CI 0.2-1.6) in the IADL domain and 2.3 (95\% CI 1.1-3.5) in the total domain. This means that particularly those patients with a more favourable HRQoL at baseline are predicted to show the largest decrease in HRQoL after $1 \mathrm{yr}$. In the SGRQ impacts domain, the intermediate-severity subgroup is also predicted to increase by over four points in $1 \mathrm{yr}$. In the GARS ADL domain, all subgroups are predicted to deteriorate statistically significant over 1 yr.

\section{DISCUSSION}

We found that COPD patients with GOLD stage IV disease experience low quality of life and functional status that deteriorate slightly over $\geqslant 1 \mathrm{yr}$. For the most part, patients who entered the study with a more favourable HRQoL and functional status showed the largest and most clinically significant decline over time. It is, however, expected that the patients who already had a less favourable health status when entering the study also experienced a deterioration, even though this could not be measured accurately.

It may be expected that patients' quality of life deteriorates rapidly prior to death because of increasing symptom burden. Our data, however, showed a gradual decline of HRQoL and functional status over time, with no indication of a steep decline at the end of life, even in those patients who died during the study period. This indicates that no additional information about the timing of the terminal stage is gained by assessing HRQoL and functional status (using SGRQ and GARS) on a regular basis. Despite this, our data do suggest individual variation in HRQoL and functional status. These individual variations are probably due to exacerbations. A study by SEEMUNGal et al. [30] showed that, in a cohort of COPD patients with slightly better lung function (mean FEV1 $40 \%$ pred), frequent exacerbations, high levels of dyspnoea and daily wheezing were associated with worse HRQoL (also measured by SGRQ) [30]. Unfortunately, we did not collect any data on exacerbations during the study period. A gradual decline in HRQoL and functional status over time is consistent with findings reported by SPENCER et al. [23] and OGA et al. [24], who studied patients in milder stages of COPD (mean FEV1 50 and $46 \%$ pred, respectively). Both studies reported a gradual decline in health status over time. This supports our hypothesis that the health status of patients with the least favourable health status will continue to deteriorate, even though our measuring instruments could not capture this deterioration adequately.

\section{Strengths and weaknesses}

We managed to include 82 patients with GOLD stage IV COPD who experienced severe limitations in daily life. Despite their limitations, they were willing and able to fill in questionnaires about their HRQoL and functional status every 3 months. There is a possibility that only patients with a more favourable health status were able to participate in this study, thus 

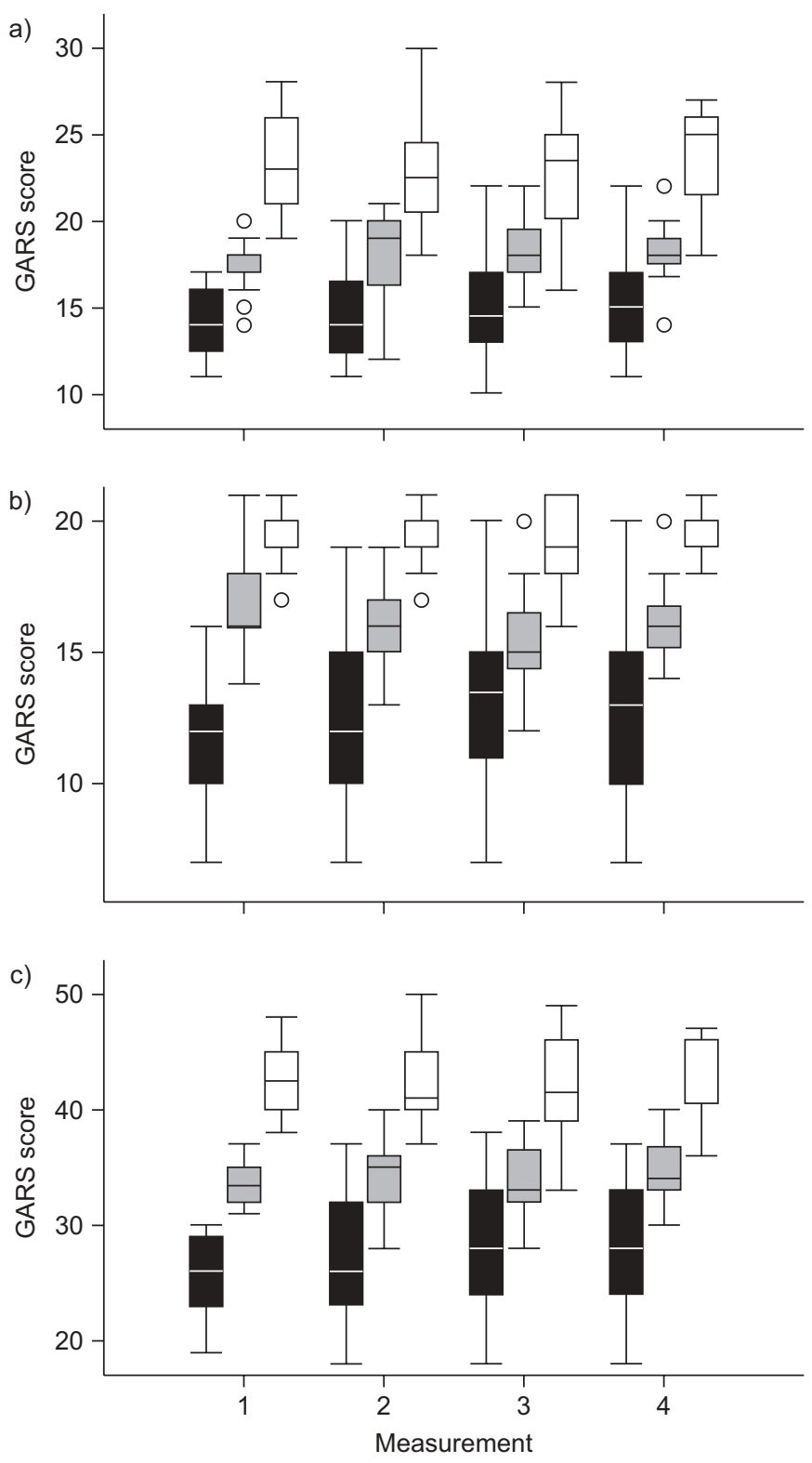

FIGURE 3. Boxplots of the a) activities of daily living (ADL), b) instrumental ADL and c) total domains of the Groningen Activities of Daily Living Restriction Scale (GARS), at 0-9 months after data entry, by severity subgroups (based on total GARS score at baseline). Boxes represent the interquartile range and whiskers

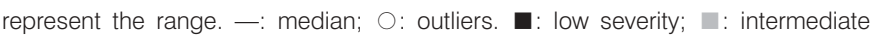
severity; $\square$ : high severity.

excluding patients with lower quality of life. We have no indication that the patients we included in our study had a health status that deviated from that generally described in GOLD stage IV patients, but even if this were the case, it would mean that the results of our study would be biased towards more favourable outcomes. The decline in HRQoL and functional status would, perhaps, be even worse if we had failed to include patients with least favourable health status. Since we had no control group, there is a possibility that our results over time are subject to some regression to the mean. However, by stratifying the scores at baseline, we were able to
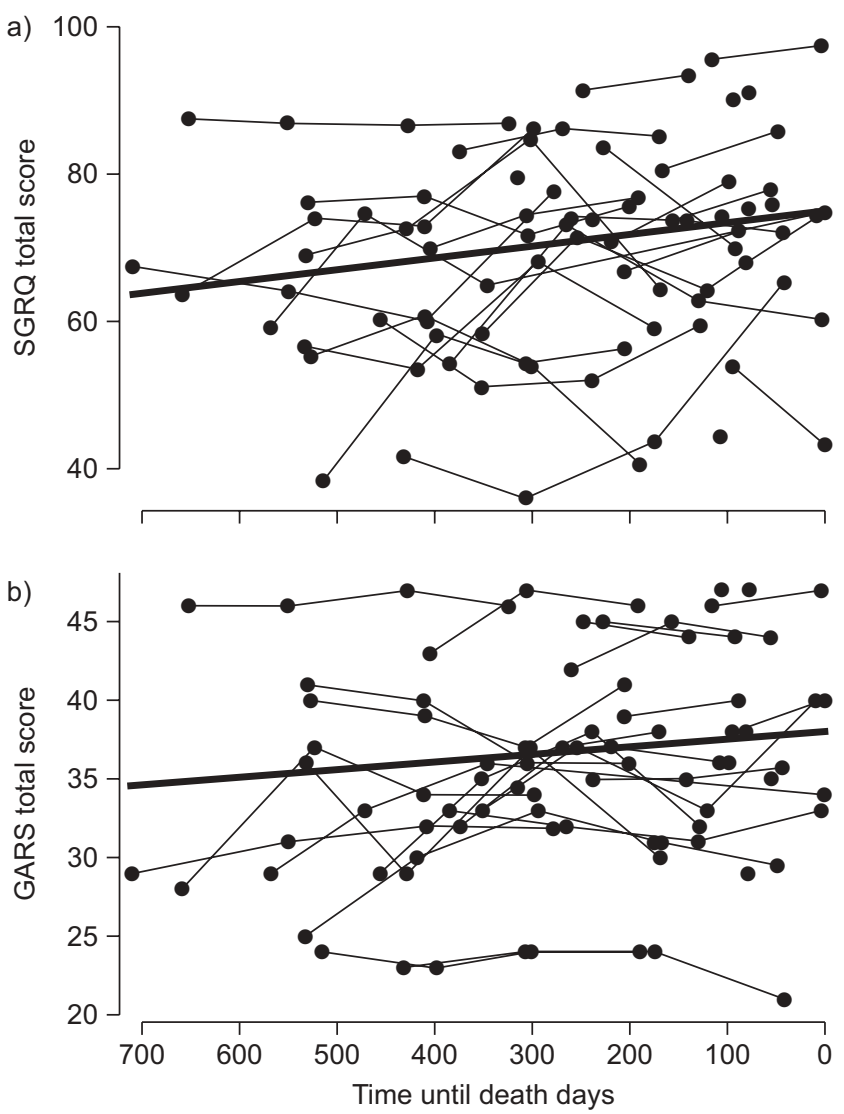

FIGURE 4. Individual measurements (-) and general trends of total a) St George's Respiratory Questionnaire (SGRQ) and b) Groningen Activities of Daily Living Restriction Scale (GARS) scores for patients who died within 2 yrs of the start of the study $(n=32)$.

examine the results in different strata. These strata continued to describe the same patterns. For instance, the mean in the low-severity subgroup remained well below the means of the intermediate- and high-severity subgroups. This makes regression to the mean very unlikely.

The patients we recruited had an FEV1 $<30 \%$ pred, a marker for GOLD stage IV. Our study criteria did not include restrictions on how long patients already had an FEV1 $<30 \%$ pred. Inclusion in the study was not marked by other additional special circumstances, such as admission to hospital or start of long-term oxygen therapy. This resulted in a mixed study population including both patients who were recently diagnosed as stage IV and patients who had already been in this stage for years. We accounted for this possible difference in disease severity by stratifying the patient population at baseline.

The HRQoL and functional status measurements used in this study were self-reported, written questionnaires and were, by definition, subjective measurements. The results show that the activity domain of the SGRQ was not appropriate for this population because the results of this domain showed a ceiling effect. Although we saw no such effect in any of the other domains of the SGRQ and GARS, the upper limits of the confidence intervals of the least favourable groups were close 

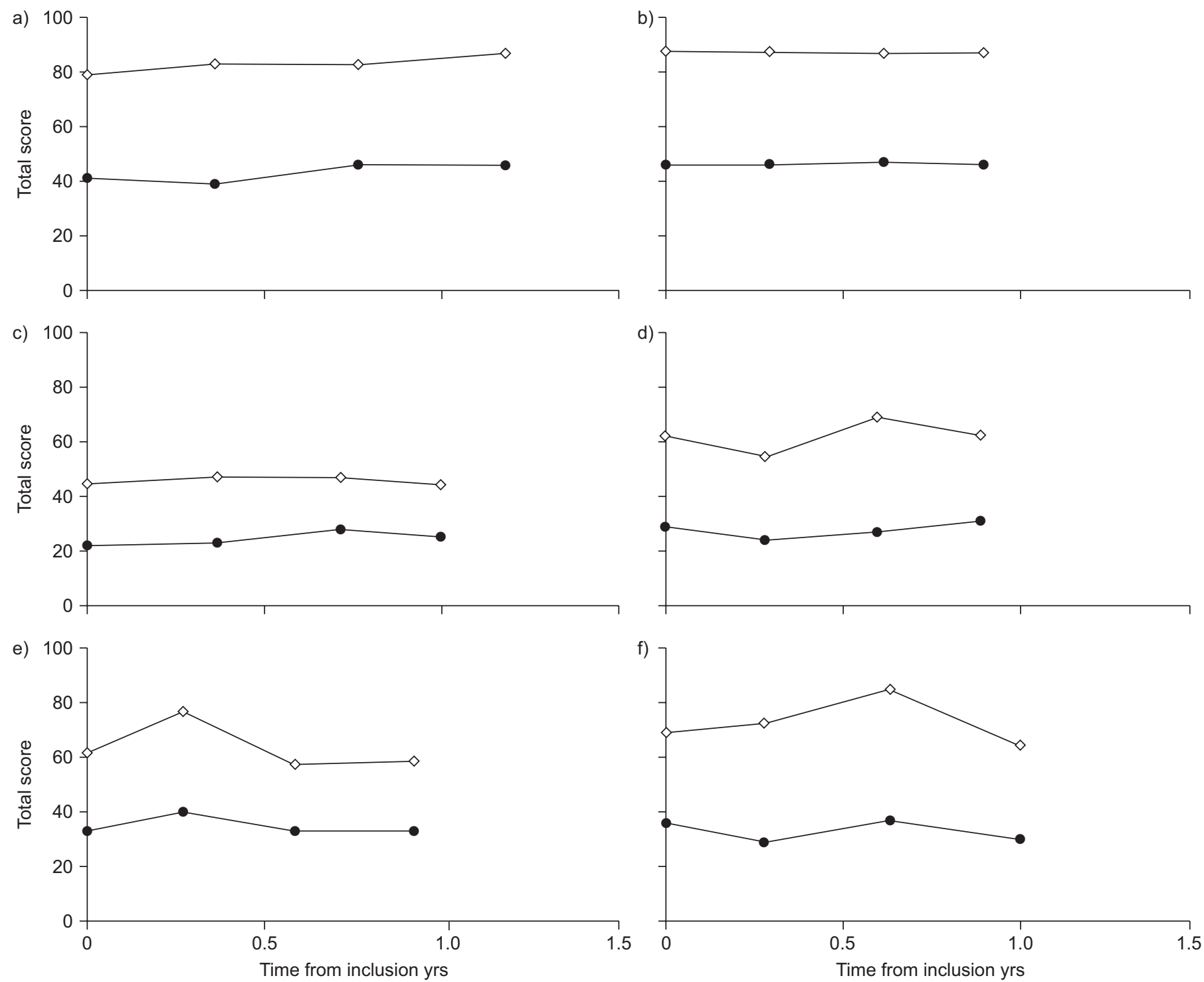

FIGURE 5. Longitudinal plots of St George's Respiratory Questionnaire

) and Groningen Activities of Daily Living Restriction Scale $(\diamond)$ scores for six individual patients.

to the maximal scores. This may be an indication that these questionnaires are not capable of monitoring further deteriorations in health status in this particular group of patients. The validity of the SGRQ in the most severe patients has been questioned before, by the developer of the SGRQ himself [31]. We would, therefore, recommend further research into the construction of a more appropriate questionnaire that is capable of detecting small changes in health status in this severely impaired group of patients. Progress in this area has already been made by the recent development of new HRQoL and ADL measures for use in this severe patient group, such as the London Chest Activity of Daily Living [32] and Maugeri Respiratory Failure (MRF)-28 [33] scales, although the latter is not designed for COPD patients specifically. Since qualitative studies have shown that patients in the end stage of COPD report a continuous deterioration in health status [17, 18, 34-36], it should also be possible to quantitatively identify the small deteriorations that the most severe patients perceive in their health status. This information may then be used to help healthcare professionals to detect deteriorations at an earlier stage and, ultimately, improve palliative care provision in endstage COPD patients.

In conclusion, the present study shows that patients with advanced COPD experience low HRQoL and functional status for a long period of time. Deterioration in health status over time was mostly seen in the group of patients who entered the study with a more favourable health status, although it is also expected that the more severe patients experience a deterioration, even though this could not be measured accurately. There was no sudden deterioration in health status visible, even in patients who died during the study period. This means that clinicians do not gain much additional support in differentiating the end stage of COPD by looking at HRQoL and functional status using the SGRQ and GARS, both widely used HRQoL and functional status measurements. 


\begin{tabular}{|c|c|c|c|}
\hline TABLE 3 & \multicolumn{3}{|c|}{$\begin{array}{l}\text { Overview of intercepts and predicted 1-yr } \\
\text { increase using longitudinal models in all } \\
\text { domains of St George's Respiratory } \\
\text { Questionnaire (SGRQ) and Groningen Activities } \\
\text { of Daily Living Restriction Scale (GARS) for all } \\
\text { severity subgroups separately }\end{array}$} \\
\hline \multicolumn{2}{|c|}{$\begin{array}{l}\text { Subgroups based on } \\
\text { total scores at baseline }\end{array}$} & Intercept $(95 \% \mathrm{Cl})$ & $\begin{array}{c}\text { Predicted 1-yr } \\
\text { increase }(95 \% \mathrm{Cl})\end{array}$ \\
\hline \multicolumn{4}{|l|}{ SGRQ } \\
\hline \multicolumn{4}{|l|}{ Symptoms } \\
\hline Low & & $45.4(39.3-51.5)$ & $3.7(-2.9-10.3)$ \\
\hline Intermedi & & $59.9(56.0-63.7)$ & $3.1(-1.6-7.8)$ \\
\hline High & & $74.3(68.3-80.4)$ & $2.4(-5.8-10.6)$ \\
\hline \multicolumn{4}{|l|}{ Activity } \\
\hline Low & & $73.3(70.2-76.5)$ & $5.6(2.5-8.8)$ \\
\hline Intermedi & & $84.1(82.1-86.1)$ & $2.9(0.5-5.2)$ \\
\hline High & & $95.0(91.8-98.1)$ & $0.1(-4.0-4.2)$ \\
\hline \multicolumn{4}{|l|}{ Impacts } \\
\hline Low & & 36.6 (33.3-39.9) & $7.0(3.0-11.1)$ \\
\hline Intermedi & & $56.2(54.1-58.3)$ & $4.5(1.5-7.5)$ \\
\hline High & & 75.9 (72.5-79.2) & $1.9(-3.4-7.3)$ \\
\hline \multicolumn{4}{|l|}{ Total } \\
\hline Low & & $49.6(46.8-52.3)$ & $6.0(3.2-8.8)$ \\
\hline Intermedi & & $65.5(63.8-67.2)$ & $3.8(1.7-5.9)$ \\
\hline High & & $81.4(78.7-84.2)$ & $1.6(-2.2-5.3)$ \\
\hline \multicolumn{4}{|l|}{ GARS } \\
\hline \multicolumn{4}{|l|}{ ADL } \\
\hline Low & & $13.9(13.1-14.7)$ & $1.0(0.2-1.9)$ \\
\hline Intermedi & & $18.3(17.8-18.8)$ & $1.0(0.4-1.6)$ \\
\hline High & & $22.7(22.0-23.4)$ & $1.1(0.1-2.0)$ \\
\hline \multicolumn{4}{|l|}{ IADL } \\
\hline Low & & $12.2(11.6-12.8)$ & $0.9(0.2-1.6)$ \\
\hline Intermedi & & $15.9(15.5-16.3)$ & $0.4(-0.1-0.8)$ \\
\hline High & & $19.6(19.0-20.1)$ & $-0.2(-1.0-0.6)$ \\
\hline \multicolumn{4}{|l|}{ Total } \\
\hline Low & & $26.1(25.0-27.2)$ & $2.3(1.1-3.5)$ \\
\hline Intermedi & & $34.2(33.5-34.9)$ & $1.7(0.8-2.5)$ \\
\hline High & & $42.2(41.1-43.3)$ & $1.1(-0.3-2.5)$ \\
\hline
\end{tabular}

ADL: activities of daily living; IADL: instrument ADL.

\section{SUPPORT STATEMENT}

The study was supported by the Netherlands Asthma Foundation (3.4.02.21).

\section{STATEMENT OF INTEREST}

None declared.

\section{ACKNOWLEDGEMENTS}

The authors would like to thank all participating patients and doctors for their time and efforts.

\section{REFERENCES}

1 Lopez AD, Mathers CD, Ezzati M, et al. Global and regional burden of disease and risk factors, 2001: systematic analysis of population health data. Lancet 2006; 367: 1747-1757.
2 World Health Organization. The Global Burden Of Disease: 2004 Update. Geneva, WHO, 2008.

3 Viegi G, Pistelli F, Sherrill DL, et al. Definition, epidemiology and natural history of COPD. Eur Respir J 2007; 30: 993-1013.

4 Murray SA, Kendall M, Boyd K, et al. Illness trajectories and palliative care. BMJ 2005; 330: 1007-1011.

5 Hansen-Flaschen J. Chronic obstructive pulmonary disease: the last year of life. Respir Care 2004; 49: 90-97.

6 Coventry PA, Grande GE, Richards DA, et al. Prediction of appropriate timing of palliative care for older adults with nonmalignant life-threatening disease: a systematic review. Age Ageing 2005; 34: 218-227.

7 Curtis JR, Engelberg RA, Wenrich MD, et al. Communication about palliative care for patients with chronic obstructive pulmonary disease. J Palliat Care 2005; 21: 157-164.

8 Knauft E, Nielsen EL, Engelberg RA, et al. Barriers and facilitators to end-of-life care communication for patients with COPD. Chest 2005; 127: 2188-2196.

9 Dean MM. End-of-life care for COPD patients. Prim Care Respir J 2008; 17: 46-50.

10 Elkington $\mathrm{H}$, White $\mathrm{P}$, Addington-Hall J, et al. The healthcare needs of chronic obstructive pulmonary disease patients in the last year of life. Palliat Med 2005; 19: 485-491.

11 Edmonds P, Karlsen S, Khan S, et al. A comparison of the palliative care needs of patients dying from chronic respiratory diseases and lung cancer. Palliat Med 2001; 15: 287-295.

12 Jones I, Kirby A, Ormiston P, et al. The needs of patients dying of chronic obstructive pulmonary disease in the community. Fam Pract 2004; 21: 310-313.

13 Rocker GM, Sinuff T, Horton R, et al. Advanced chronic obstructive pulmonary disease: innovative approaches to palliation. J Palliat Med 2007; 10: 783-797.

14 Elkington $\mathrm{H}$, White $\mathrm{P}$, Addington-Hall J, et al. The last year of life of COPD: a qualitative study of symptoms and services. Respir Med 2004; 98: 439-445.

15 Bailey PH. The dyspnea-anxiety-dyspnea cycle - COPD patients' stories of breathlessness: "it's scary/when you can't breathe". Qual Health Res 2004; 14: 760-778.

16 Curtis JR. Palliative and end-of-life care for patients with severe COPD. Eur Respir J 2008; 32: 796-803.

17 Ek K, Ternestedt BM. Living with chronic obstructive pulmonary disease at the end of life: a phenomenological study. J Adv Nurs 2008; 62: 470-478.

18 Fitzsimons D, Mullan D, Wilson JS, et al. The challenge of patients unmet palliative care needs in the final stages of chronic illness. Palliat Med 2007; 21: 313-322.

19 Gore JM, Brophy CJ, Greenstone MA. How well do we care for patients with end stage chronic obstructive pulmonary disease (COPD)? A comparison of palliative care and quality of life in COPD and lung cancer. Thorax 2000; 55: 1000-1006.

20 Claessens MT, Lynn J, Zhong Z, et al. Dying with lung cancer or chronic obstructive pulmonary disease: insights from SUPPORT. Study to Understand Prognoses and Preferences for Outcomes and Risks of Treatments. J Am Geriatr Soc 2000; 48: S146-S153.

21 Habraken JM, ter Riet G, Gore JM, et al. Health-related quality of life in end-stage COPD and lung cancer patients. J Pain Symptom Manage 2009; 37: 973-981.

22 Varkey B. Unfulfilled palliative care needs of chronic obstructive pulmonary disease patients. Curr Opin Pulm Med 2006; 12: 103-105.

23 Spencer S, Calverley PM, Sherwood Burge P, et al. Health status deterioration in patients with chronic obstructive pulmonary disease. Am J Respir Crit Care Med 2001; 163: 122-128.

24 Oga $T$, Nishimura $K$, Tsukino $M$, et al. Longitudinal deteriorations in patient reported outcomes in patients with COPD. Resp Med 2007; 101: 146-153. 
25 Global Initiative for Chronic Obstructive Lung Disease. Global Strategy for the Diagnosis, Management, and Prevention of COPD. http: / / goldcopd.com/Guidelineitem.asp?11=2\&12=1\&intId=2003 Date last accessed: 2006. Date last updated: 2009.

26 Jones PW, Quirk FH, Baveystock CM, et al. A self-complete measure of health status for chronic airflow limitation. The St. George's Respiratory Questionnaire. Am Rev Respir Dis 1992; 145: 1321-1327.

27 Jones PW. Interpreting thresholds for a clinically significant change in health status in asthma and COPD. Eur Respir J 2002; 19: 398-404.

28 Kempen GI, Miedema I, Ormel J, et al. The assessment of disability with the Groningen Activity Restriction Scale. Conceptual framework and psychometric properties. Soc Sci Med 1996; 43: 1601-1610.

29 Fletcher CM, Elmes PC, Fairbairn AS, et al. The significance of respiratory symptoms and the diagnosis of chronic bronchitis in a working population. Br Med J 1959; 2: 257-266.

30 Seemungal TA, Donaldson GC, Paul EA, et al. Effect of exacerbation on quality of life in patients with chronic obstructive pulmonary disease. Am J Respir Crit Care Med 1998; 157: 1418-1422.
31 Jones PW. Health status measurement in chronic obstructive pulmonary disease. Thorax 2001; 56: 880-887.

32 Garrod R, Bestall JC, Paul EA, et al. Development and validation of a standardized measure of activity of daily living in patients with severe COPD: the London Chest Activity of Daily Living scale (LCADL). Respir Med 2000; 94: 589-596.

33 Carone M, Bertolotti G, Anchisi F, et al. Analysis of factors that characterize health impairment in patients with chronic respiratory failure. Quality of life in chronic respiratory failure group. Eur Respir J 1999; 13: 1293-1300.

34 Cicutto L, Brooks D, Henderson K. Self-care issues from the perspective of individuals with chronic obstructive pulmonary disease. Patient Educ Couns 2004; 55: 168-176.

35 Habraken JM, Pols J, Bindels PJ, et al. The silence of patients with end-stage COPD: a qualitative study. Br J Gen Pract 2008; 58: 844-849.

36 Seamark DA, Blake SD, Seamark CJ, et al. Living with severe chronic obstructive pulmonary disease (COPD): perceptions of patients and their carers. An interpretative phenomenological analysis. Palliat Med 2004; 18: 619-625. 